\title{
$\operatorname{Con}-9410131--8$ \\ $\operatorname{sAn} 93.4074 \mathrm{C}$
}

\section{A COMPARISON OF PHASE CHANGE PHENOMENA IN CTH WITH EXPERIMENTAL DATA}

\author{
E. S. Hertel, Jr., ${ }^{1}$ R. L. McIntosh, ${ }^{1}$ and Bruce C. Patterson ${ }^{2}$ \\ 1: Sandia National Laboratories, Albuquerque, New Mexico \\ 2: Wright Laboratory/Armament Directorate, Eglin AFB, FL
}

\begin{abstract}
Summary-An estimate of the current state of the predictive capabilities of hydrocodes for impacts where phase changes may be important was made by simulating a series of experiments where zinc spheres impact thin zinc plates at 4 velocities. The experimental data was taken from Mullin, et al. [1] and consisted of radiographs of the debris cloud and estimates of the momentum in the debris cloud along the velocity vector. The velocities ranged from 3.30 to $6.79 \mathrm{~km} / \mathrm{s}$ indicating that the debris consists of solid/molten fragments to predominately vapor phase material as the velocity increases. The simulations reveal that the CTH hydro can accurately predict the debris structure and momentum transfer for this class of impacts.
\end{abstract}

\section{INTRODUCTION}

It has been recognized that the use of shock physics analysis packages (aka, hydrocodes) in the impact regime where materials can be expected to change phase is strongly dependent on the ability of the hydrocode to accurately model the partitioning of energy and volume change as a result of the pressure induced on impact [2]. The functional form of energy and volume change is commonly referred to as the equationof-state (EOS) of a material. Other features of the hydrocode, such as the accuracy of the numerical schemes, the ability to model material deformation due to various loading schemes, and failure and fragmentation, are also important, but a key feature is still the decision on how to partition energy and volume change. The EOS of a material can be expressed in terms of an analytic expression or tabular representation of the complex surface. Both methods rely on a combination of theory and experimental data to build the surface representation. The experiments are typically simple flat plate impacts (one-dimensional) that concentrate on complete understanding of a few features. The EOS experiments are useful in understanding the underlying phenomenology of the material, but generally are not useful in validating multi-dimensional features of the hydrocodes. Other experiments that exploit their multi-dimensional characteristics are needed to validate the numerics and physics that are important in conjunction with the EOS experiments.

It is useful to discuss some of the concerns and features surrounding the need for validation experiments in terms of a canonical problem. The canonical problem was chosen to illustrate interactions typical of a projectile striking a complex target. From the previous discussion, it should be clear that code validation requires relatively simple (but carefully chosen) experiments that reflect the key physical processes of the actual (but complex) interactions.

Figure 1 shows a sketch of a suggested canonical problem for code validation over a range of impact velocities along with diagnostic equipment appropriate for such a problem. This sketch shows a projectile (shown here as a sphere, but any simple shape could be used) impacting a plate. The initial impact will produce a debris cloud whose state will depend on the impact velocity and material characteristics. The debris cloud will then traverse a void space and another impact will occur with a second plate. The secondary impact will also produce a debris cloud whose state will now depend on the characteristics of the initial debris cloud, the secondary impact velocity, and the material characteristics. It should be expected that the secondary debris cloud will have different characteristics than the first cloud. The secondary debris cloud

will also traverse a void space and impact a third plate.
A key aspect of impact induced fragmentation is that as the velocity increases, the size distribution of the resulting debris cloud changes. This idea is shown in Figure 2 in a qualitative sense. At relatively low 


\section{DISCLAIMER}

This report was prepared as an account of work sponsored by an agency of the United States Government. Neither the United States Government nor any agency thereof, nor any of their employees, make any warranty, express or implied, or assumes any legal liability or responsibility for the accuracy, completeness, or usefulness of any information, apparatus, product, or process disclosed, or represents that its use would not infringe privately owned rights. Reference herein to any specific commercial product, process, or service by trade name, trademark, manufacturer, or otherwise does not necessarily constitute or imply its endorsement, recommendation, or favoring by the United States Government or any agency thereof. The views and opinions of authors expressed herein do not necessarily state or reflect those of the United States Government or any agency thereof. 


\section{DISCLAIMER}

Portions of this document may be illegible in electronic image products. Images are produced from the best available original document. 
Fig. 1. Canonical problem description.

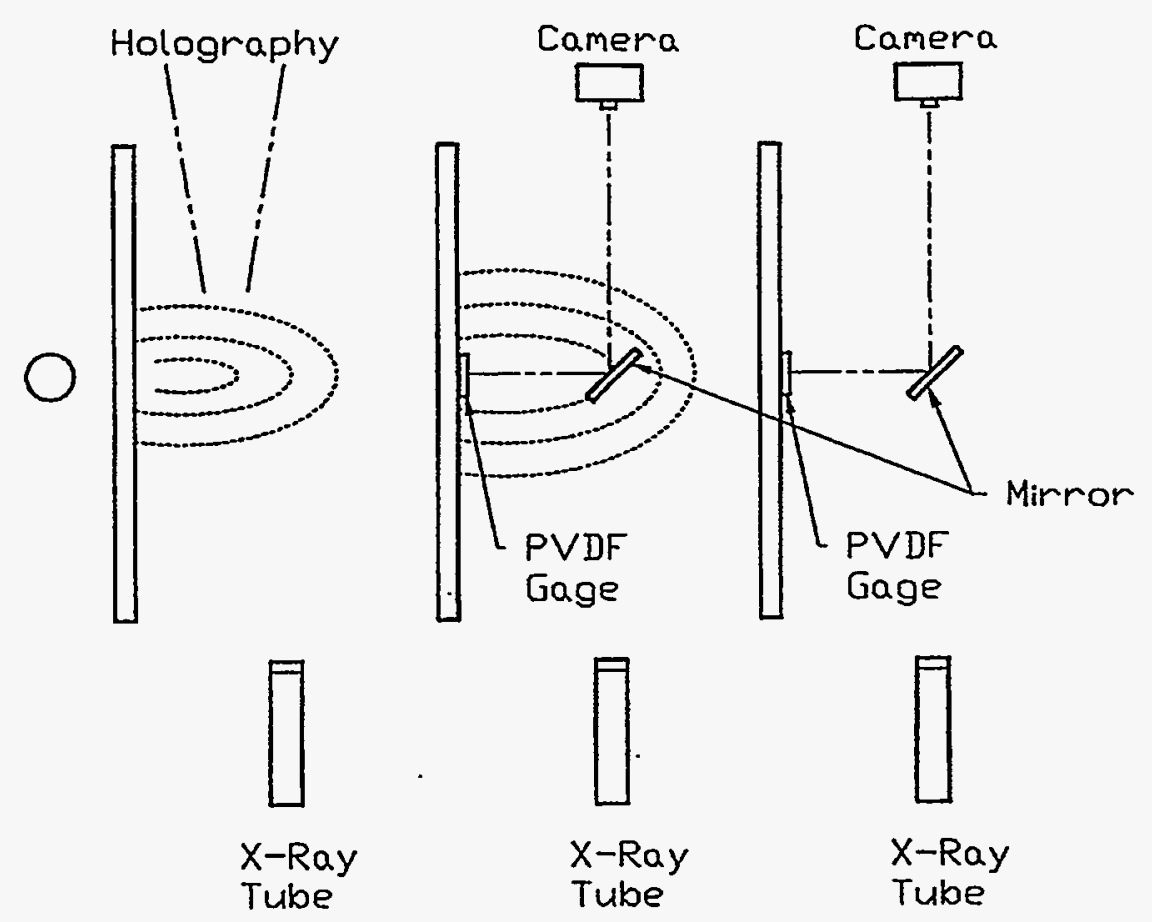

Fig. 2. Fragment distribution.

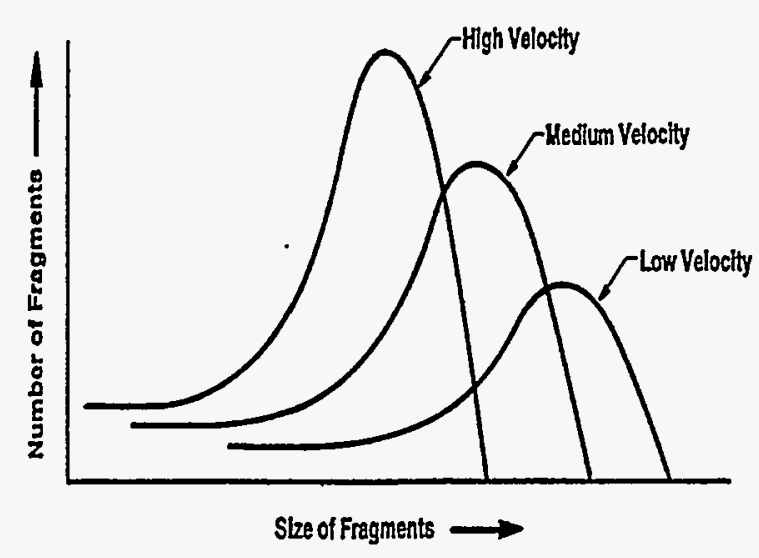

Fig. 3. Low velocity impact debris cloud.

impact velocities, the size distribution is skewed towards a few large particles with no very small particles. As the velocity increases, the number of large particles decreases and the number of small to very small particles increases. The size of the largest particle also decreases as the total number of particles increases. This progression has interesting ramifications for our canonical problem as is illustrated by the following:

At low impact velocities, a few large fragments are created by the initial impact. This scenario can be visualized in Figure 3. Here, large fragments refer to objects much larger (fully resolved) than the computational mesh. The dynamics of the initial impact are dominated by tensile failure phenomena. The secondary impacts are characterized by discrete impacts with little or no synergism. A smaller number of spall generated fragments may be ejected from the back of the second plate. The tertiary impacts are very few and damage at the third plate is dominated by strength rather than hydrodynamics. As the velocity increases, the initial debris cloud is characterized by more and smaller fragments. This scenario can be visualized in Figure 4. Here, the fragments are not larger than the computational mesh, but comparable in size (marginally resolved). The failure regime is still dominated by tensile phenomena, but the process is 
Fig. 4. Medium velocity impact debris cloud.

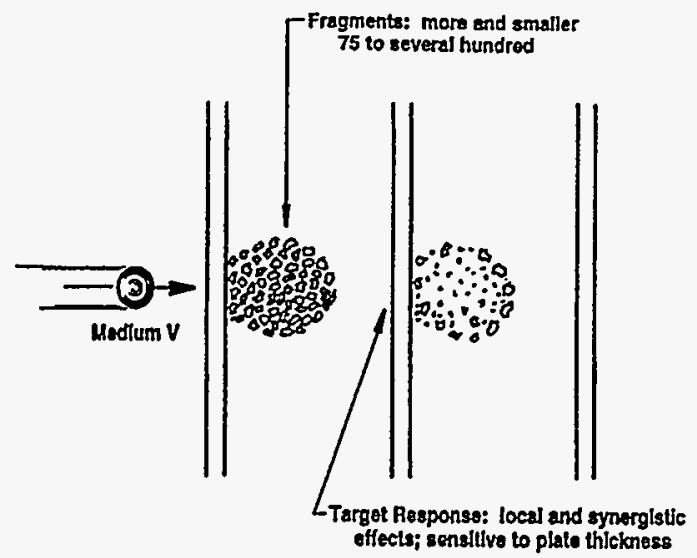

Fig. 5. High velocity impact debris cloud.

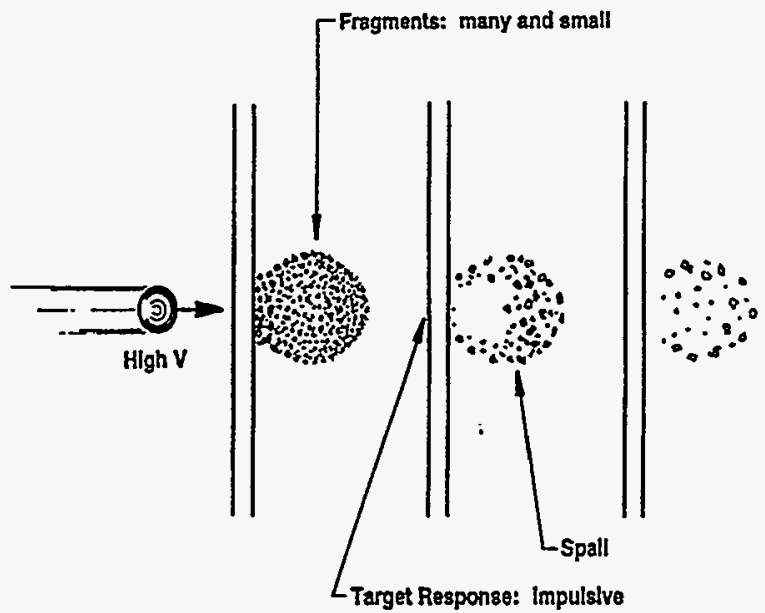

statistically determined and only averages of size and trajectory have meaning. Depending on material characteristic, the initial debris cloud may contain some melted material along with discrete particles. The secondary target response is determined by a combination of local and synergistic effects. The secondary debris cloud may consist of fewer and larger spall-induced fragments due to the discrete impacts mixed with particles that survived perforation, or a spall plug due to impulsive loading. Loading on the third plate now resembles secondary loading for a lower velocity initial impact. With a further increase in velocity, the initial debris cloud is characterized by many very small fragments. This scenario can be visualized in Figure 5. Here, the fragments are much smaller than the computational mesh and are being treated as a continuum of material by the hydrocodes. The failure regime is dominated by tensile phenomena, but the process is statistically determined and only distribution and shape of the debris cloud have meaning. Depending on material characteristic, the initial debris cloud will contain some melted material, some vapor, and some solid particles. The secondary target response is principally determined by impulsive loading. The secondary debris cloud will consist of more and smaller fragments and should be similar to the initial debris cloud of a medium velocity impact. Loading on the third plate now resembles secondary loading for a medium velocity initial impact.

Table 1. Experimental series initial conditions

\begin{tabular}{|c|c|c|c|c|c|}
\hline Test & $\begin{array}{c}\text { Impact Velocity } \\
(\mathrm{km} / \mathrm{s})\end{array}$ & $\begin{array}{c}\text { Projectile Diameter } \\
(\mathrm{mm})\end{array}$ & $\begin{array}{c}\text { Witness Thickness } \\
(\mathrm{mm})\end{array}$ & $\begin{array}{c}\text { Bumper Thickness } \\
(\mathrm{mm})\end{array}$ & $\begin{array}{c}\text { Plate Separation } \\
(\mathrm{mm})\end{array}$ \\
\hline \hline 8 & 3.30 & 11.074 & 25.4 & 5.131 & 220.0 \\
\hline 9 & 5.08 & 11.074 & 25.4 & 5.131 & 220.0 \\
\hline 10 & 6.02 & 11.074 & 25.4 & 5.131 & 220.0 \\
\hline 11 & 6.79 & 11.074 & 25.4 & 5.131 & 220.0 \\
\hline
\end{tabular}

It should be clear from the above discussion that even for high velocity initial impacts, a critical link to lower velocity phenomena is maintained for complex targets. This fact makes it clear that a relatively simple experimental series can be used to simulate many of the important features in complex higher velocity impacts. Consider an experimental series where the impact velocities and materials were chosen to produce a range of debris cloud characteristics from discrete particles to diffuse vapor. The debris clouds would impact a witness plate where diagnostic instruments were used to record key physical parameters. This series would mimic the cascade of high velocity debris to a lower velocity state with each impact event. The hydrocodes could be used to compare against the data to assess their validity to model the more complex interactions in a multi-plate impact event. The ideas contained in this discussion are described in more detail in a joint paper with Anderson [3]. 


\section{EXPERIMENTAL SERIES}

An existing series of impact experiments for zinc spheres impacting a thin zinc plate was described by Mullin, et al. [1]. This series spans an impact velocity range from 3.3 to $6.8 \mathrm{~km} / \mathrm{s}$ which gives rise to debris clouds composed of solid fragments at the lower velocities and a mixture of molten fragments and vapor at the higher velocities. This series of experimental data closely matches the needs of code validation for complex impacts as described in the previous section and provides the basis for a useful evaluation of hydrocode capabilities over the range of impact velocities where phase changes occur. The experiments consisted of measurements of the momentum of the debris cloud and ejecta along the line of flight, stress levels in the witness plate, damage to the witness plate, and debris cloud size and shape. The specifics of the experiments used in this study are contained in Table 1 . Reproductions of the experimental debris structure are contained in Figures 6 thru 9.

Fig. 6. Experimental debris cloud for Test $8(3.30 \mathrm{~km} / \mathrm{s}$ impact velocity).

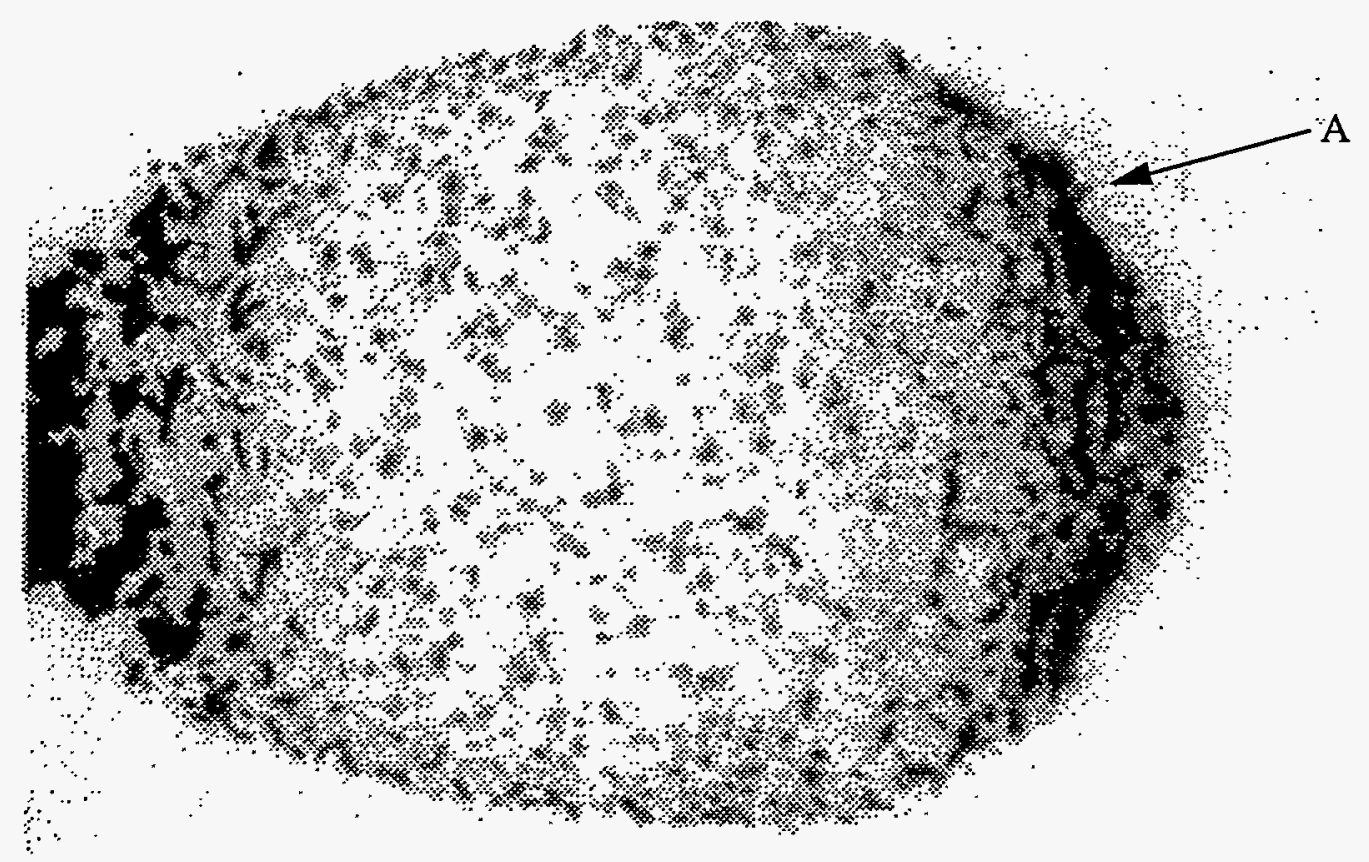

The debris cloud and momentum quantitative data for comparison purposes is contained in Table 2 . The momentum data (although with an unknown level of experimental variation) can be used to assess the accuracy of the transport models in the hydrocode. The witness plate damage characteristics are a sensitive barometer of the differential transport of debris in condensed and vapor states. The shape of the debris clouds gives insight into the partitioning of energy upon release from the shocked state. A careful examination of the entire experimental and computational record will allow an estimate of the effectiveness of hydrocodes in modelling complex interactions where phase change, debris transport, and secondary impacts are important.

\section{CTH RESULTS}

The four experiments described above were the focus of CTH [4] simulations. Each of the four experiments were simulated twice, once with very high resolution $(0.01 \times 0.01 \mathrm{~cm}$ or 55 zones across the initial projectile radius) to study the evolution of the debris cloud before impact with the witness plate; and once with lower resolution $(0.025 \times 0.025 \mathrm{~cm}$ or 22 zones across the initial projectile radius) to study the entire experiment. The lower resolution used for half of the simulations is still a much higher resolution than is considered minimal (4 zones across the smallest dimension) and should give acceptable answers. The low resolution is also similar to other simulations of comparable problems [5,6]. Because of computational limitations, the high resolution simulations were completed with a reduced geometric and temporal domain that matched the experimental debris clouds in Figures 6 through 9. The figures display the axi-symmetric simulation reflected about the $\mathrm{x}=0$ axis. The right side of each image displays a simulated radiograph where the density is summed along the line of sight and plotted with a grey scale. The left side of each image displays the 
Fig. 7. Experimental debris cloud for Test 9 (5.08 km/s impact velocity).

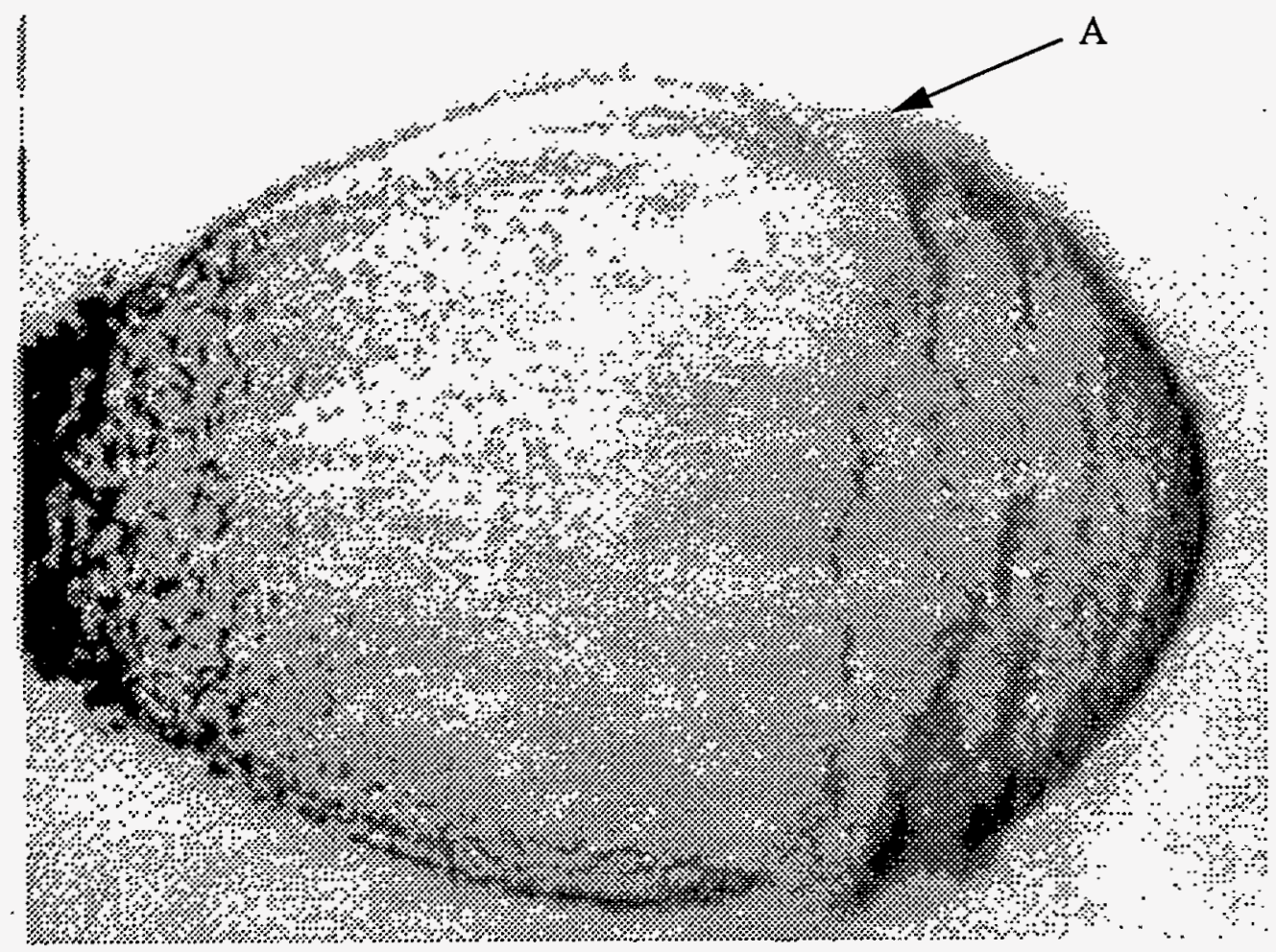

Fig. 8. Experimental debris cloud for Test 10 ( $6.02 \mathrm{~km} / \mathrm{s}$ impact velocity).

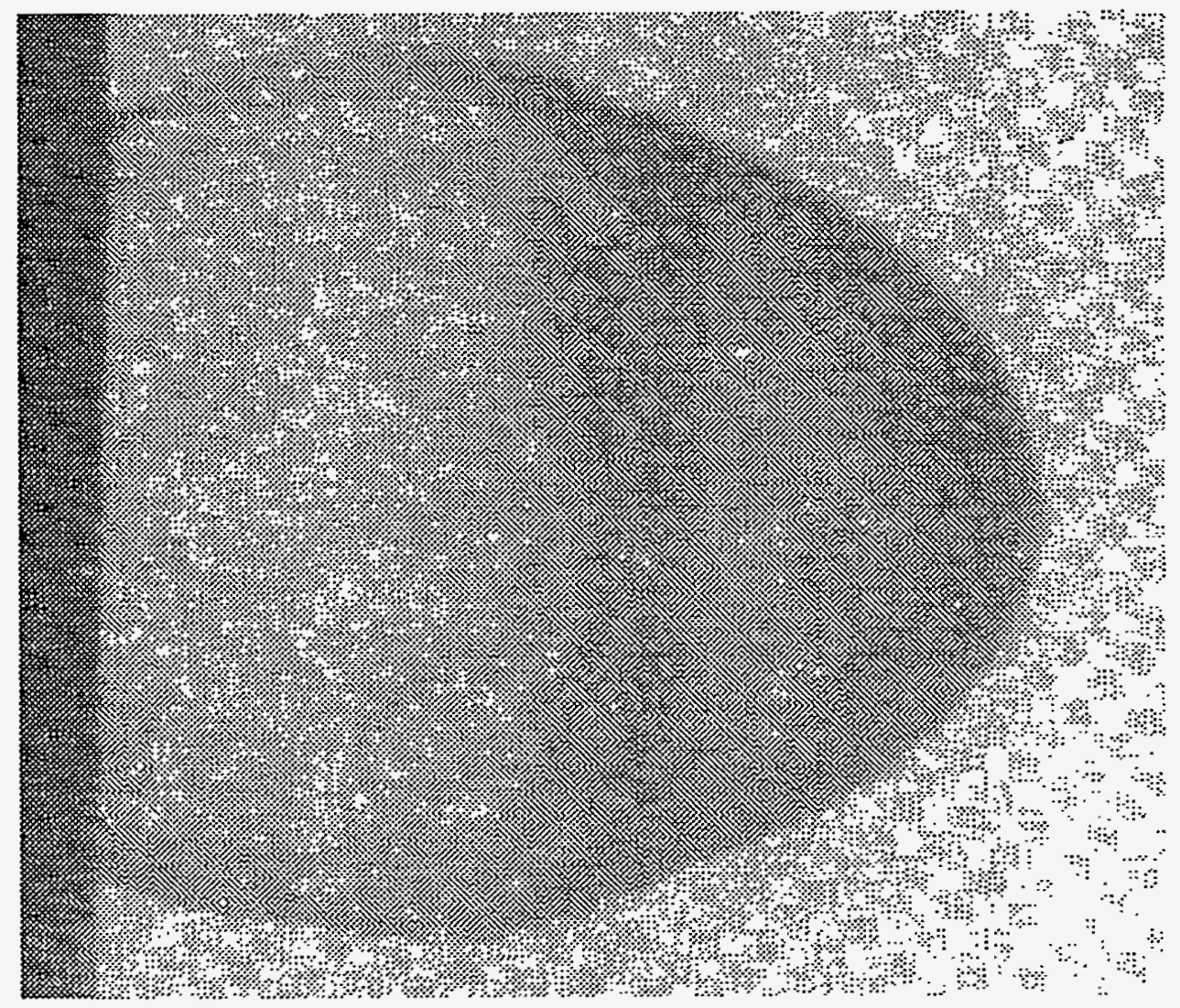

material locations where the light grey color represents the original bumper material and the dark grey represents the original projectile material. The low resolution simulations were used in the prediction of the momentum data only. The simulations used an equation-of-state that was developed for a previous study [7] of zinc vaporization that accurately matched the experimental data. The constitutive model was linearly-elastic, perfectly-plastic with a von Mises yield surface of 1.5 kbars. Failure was initiated by limiting the tensile 
Fig. 9. Experimental debris cloud for Test $11(6.79 \mathrm{~km} / \mathrm{s}$ impact velocity).

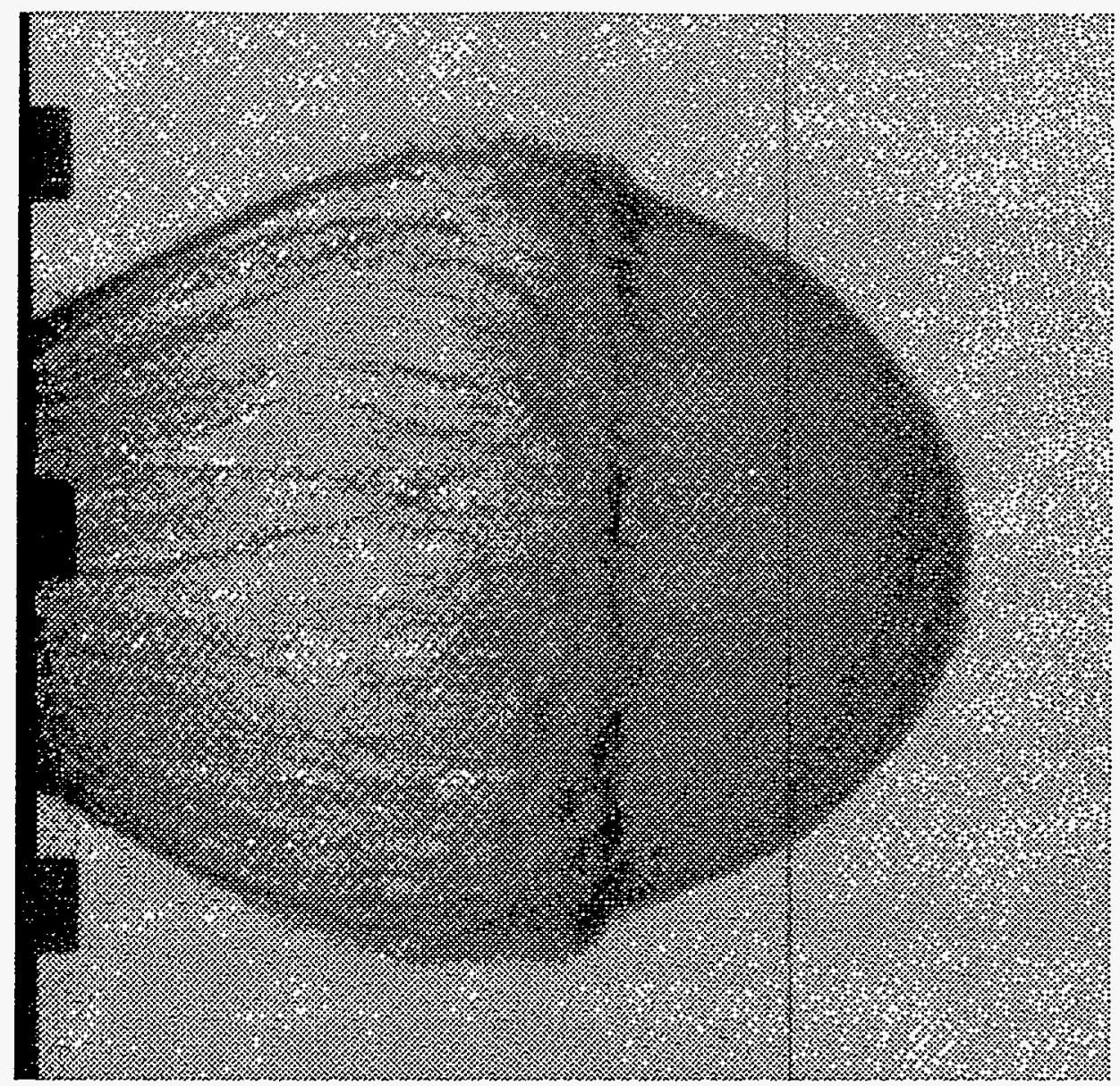

Table 2. Experimental Comparison Data

\begin{tabular}{|c|c|c|c|c|c|c|c|}
\hline Test & $\begin{array}{c}\text { Debris } \\
\text { Cloud } \\
\text { Width } \\
(\mathrm{mm})\end{array}$ & $\begin{array}{c}\text { Outer Spray } \\
\text { Diameter } \\
(\mathrm{mm})\end{array}$ & $\begin{array}{c}\text { Inner Spray } \\
\text { Diameter } \\
(\mathrm{mm})\end{array}$ & $\begin{array}{c}\text { Projectile } \\
\text { Momentum } \\
(\mathrm{gm} \mathrm{km} / \mathrm{s})\end{array}$ & $\begin{array}{c}\text { Forward Ejecta } \\
\text { Momentum } \\
(\mathrm{gm} \mathrm{km} / \mathrm{s})\end{array}$ & $\begin{array}{c}\text { Rearward } \\
\text { Ejecta } \\
\text { Momentum } \\
\text { (gm km/s) }\end{array}$ & $\begin{array}{c}\text { X-Ray } \\
\text { Time } \\
\text { (us) }\end{array}$ \\
\hline \hline 8 & 12.06 & 35.56 & 15.24 & 16.7 & 3.88 & 27.61 & 60.3 \\
\hline 9 & 14.47 & 34.29 & 11.43 & 25.8 & 9.43 & 37.06 & 45.6 \\
\hline 10 & 4.73 & 35.56 & 17.78 & 30.6 & 10.38 & 53.76 & 11.1 \\
\hline 11 & 9.78 & 35.56 & 19.05 & 34.5 & 12.33 & 54.55 & 24.5 \\
\hline
\end{tabular}

region of the equation-of-state by a $P_{\min }$ criteria of 3.0 kbars. Upon reaching the $P_{\min }$ criteria, CTH adds void to the computational cell. This relieves the tensile state and allows for the formation of cracks and fragments.

The first area for comparison is the debris cloud structure as a function of impact velocity. Figures 10 thru 13 display the high resolution debris clouds as simulated by CTH for the experiments described above. In addition to the structure comparisons, tabulated results to compare to the data in Table 2 are contained in Table 3. A comparison of the momentum data for the low resolution simulations is contained in Table 4.

\section{DISCUSSION AND CONCLUSIONS}

There are two principal areas for comparison between the experimental data and computational results, the shape and thermodynamic state of the debris cloud and the momentum transfer results. From the stand- 
Fig. 10. Simulated debris cloud for Test $8(3.30 \mathrm{~km} / \mathrm{s}$ impact velocity).

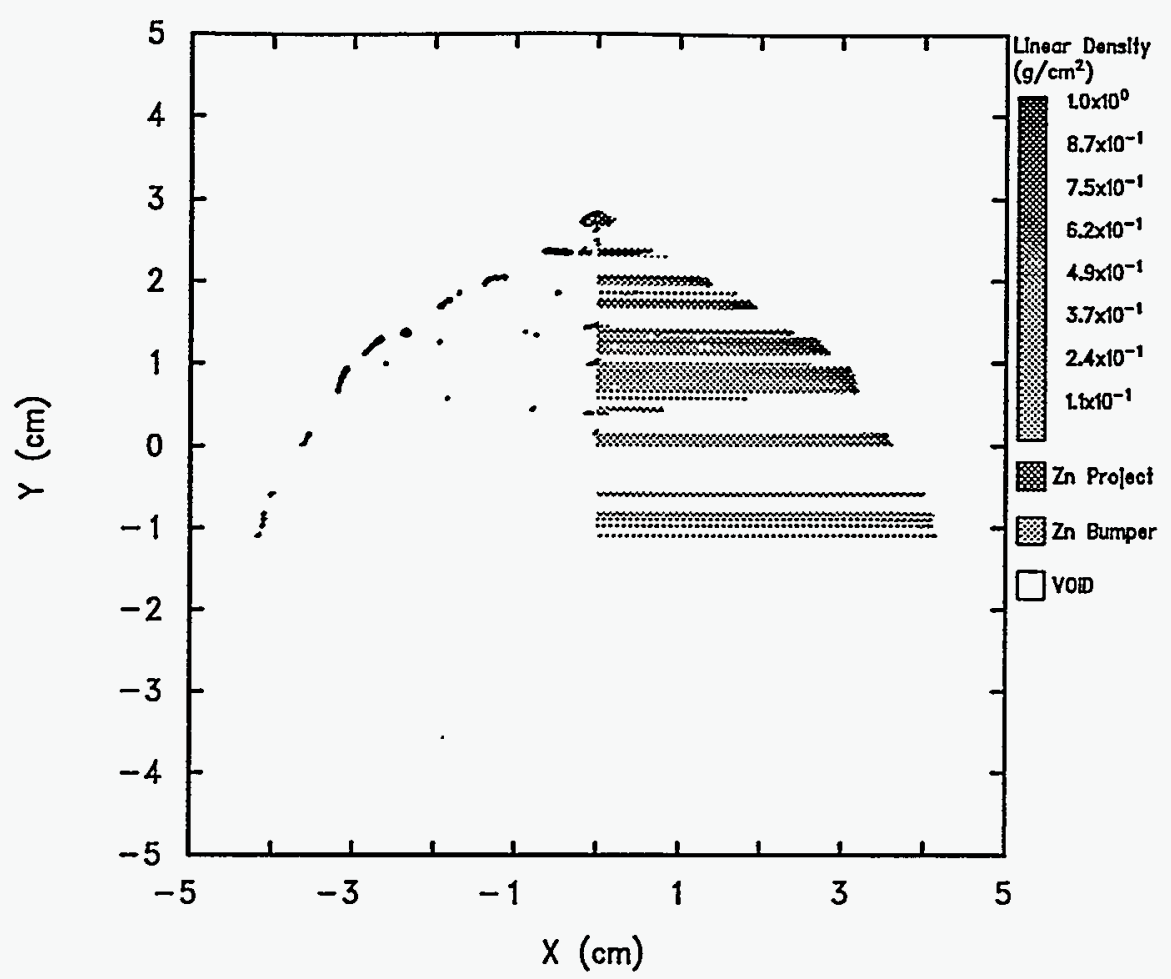

Fig. 11. Simulated debris cloud for Test $9(5.08 \mathrm{~km} / \mathrm{s}$ impact velocity).

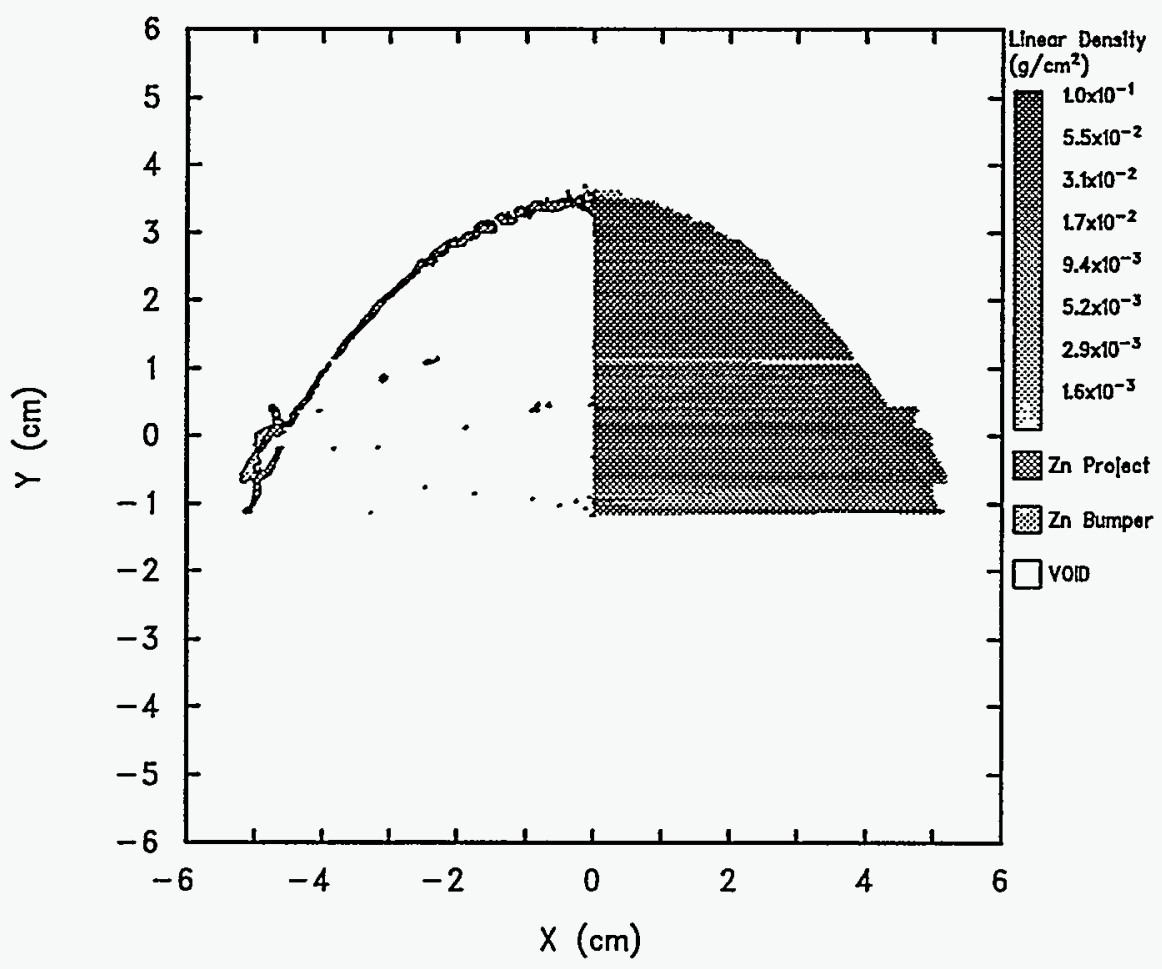

point of the physics of interactions, comparisons of the shape and thermodynamic state of the debris cloud principally test the equation-of-state and constitutive models used. In addition, there are numerical issues surrounding the ability of the code to accurately advect small fragments or vaporous material in the computational mesh that are tested by debris cloud simulations. The ability to correctly transfer momentum depends primarily on numerical issues, although, the equation-of-state and constitutive model can have an effect. Even though stress gauge comparisons were not made for this paper, the debris cloud structure, it's velocity distribution, the coupling of the debris impact into the witness plate, and the propagation of the stress waves through the witness plate after impact are critical to the accurate modelling of gauge data. The 
Fig. 12. Simulated debris cloud for Test $10(6.02 \mathrm{~km} / \mathrm{s}$ impact velocity).

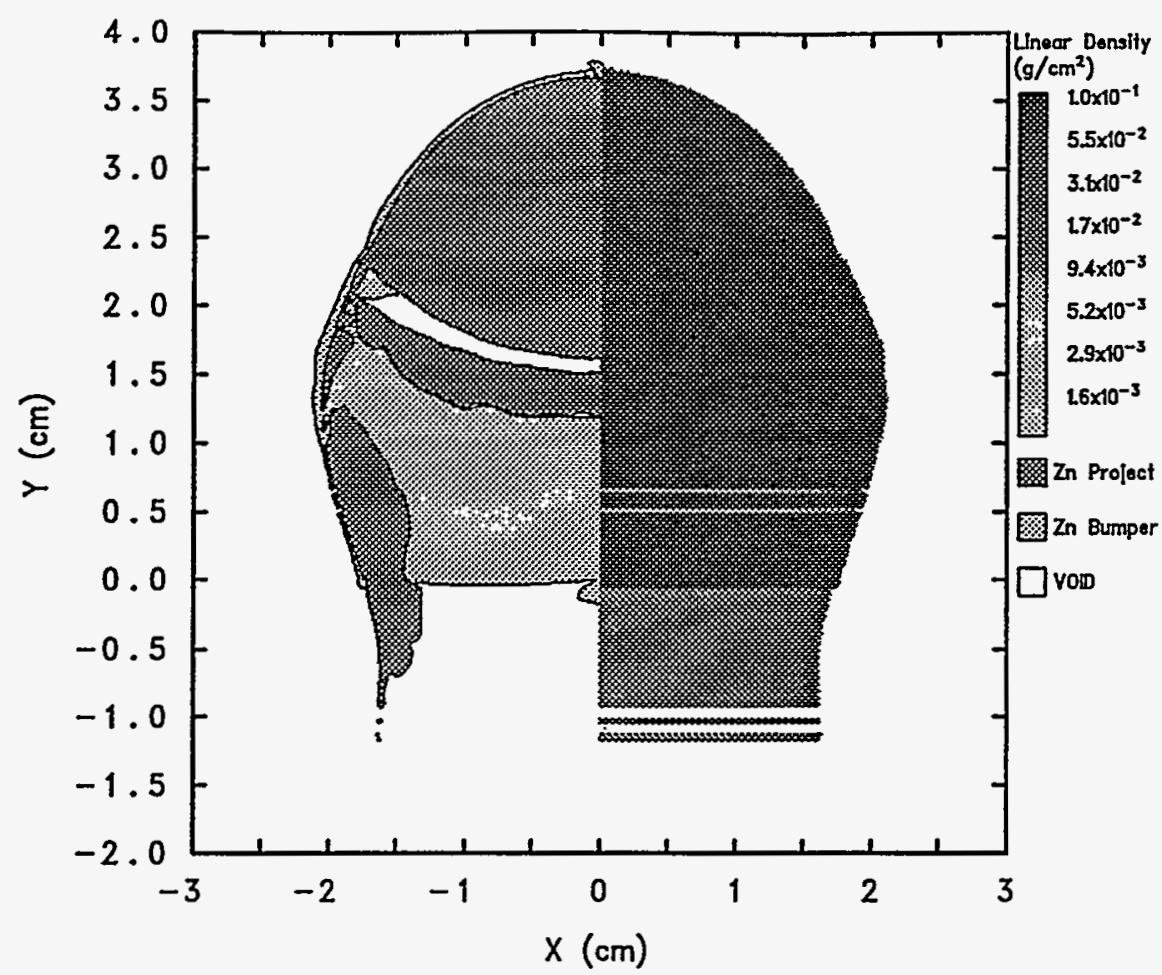

Fig. 13. Simulated debris cloud for Test $11(6.79 \mathrm{~km} / \mathrm{s}$ impact velocity).

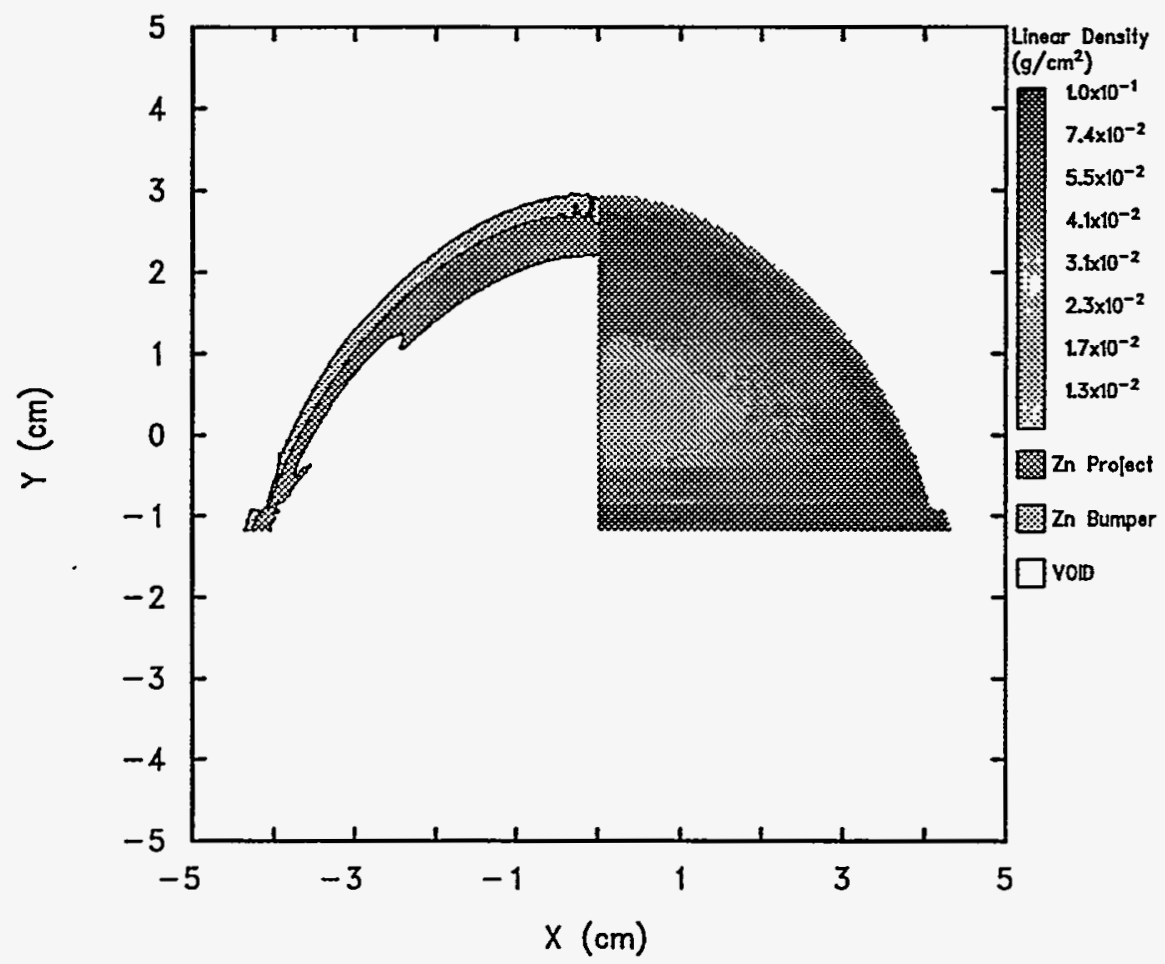

important comparisons to make are between Figures 6 and 10,7 and 11, 8 and 12, and 9 and 13. These correspond to Tests $8,9,10$, and 11 . The computational results discussed here are the high resolution prediction for the debris cloud structure and the low resolution predictions for the momentum measurements.

Test 8 (Figure 6) consists of a shell of evenly distributed debris particles that are approximately 100 times smaller than the width of the debris cloud, or $-0.08 \mathrm{~cm}$ with a high density region at the leading surface of the cloud. In addition, the leading edge of the cloud has two distinct regions (see $A$ in Figure 6 ) of different radii with the front-most $2 \mathrm{~cm}$ of the cloud having a nearly solid appearance. To visualize a fragment with CTH, the object must be larger than a computational cell. The high resolution $(0.01 \mathrm{~cm}$ zones $) \mathrm{CTH}$ simu- 
lations are capable of visualizing the typical fragment size noted in the experimental radiograph in the computational domain. The resolution is sufficient to match the dual radii seen in Figure 6. Figure 10 displays the CTH results for Test 8 in axi-symmetric geometry, where the material locations are identified by the light and dark grey coloration (dark grey corresponds to the original projectile and the light grey to the bumper plate) on the left side of the figure; the right side of the figure displays a continuous grey scale of density summed along the line of sight. This visualization technique replicates the information contained in a radiograph where material density is summed along the line of sight as if the axi-symmetric geometry was rotated about the symmetry line. Further inspection of the results reveals that the smaller radius debris bubble is composed of mixed projectile and bumper material and the remainder of the debris cloud is principally composed of projectile material. Even though the debris cloud diameter is under-predicted by CTH, the general mass distribution (in particular, the dual radius debris structure at the leading edge) is matched by the simulations. The debris cloud in Test 8 is composed of solid fragments and the prediction of fragmentation events is one area where the hydrocodes are widely considered to be deficient.

Table 3. Experimental - computational debris cloud size comparison.

\begin{tabular}{|c|c|c|c|}
\hline Test & $\begin{array}{c}\text { Experimental Debris Cloud Width } \\
(\mathrm{cm})\end{array}$ & $\begin{array}{c}\text { Computational Debris Cloud Width } \\
(\mathrm{cm})\end{array}$ & $\begin{array}{c}\text { X-Ray Time } \\
(\mu \mathrm{s})\end{array}$ \\
\hline \hline 8 & 12.06 & 8.5 & 60.3 \\
\hline 9 & 14.47 & $>10.5$ & 45.6 \\
\hline 10 & 4.73 & 4.3 & 11.1 \\
\hline 11 & 9.78 & $>8.6$ & 24.5 \\
\hline
\end{tabular}

Table 4. Experimental - computational momentum comparison data

\begin{tabular}{|c|c|c|c|c|c|c|c|c|}
\hline Test & $\begin{array}{c}\text { Target } \\
\mathrm{mv}(-) \\
(\mathrm{gm} \mathrm{km} / \mathrm{s})\end{array}$ & $\begin{array}{c}\text { Projectile } \\
\mathrm{mv}(-) \\
(\mathrm{gm} \mathrm{km} / \mathrm{s})\end{array}$ & $\begin{array}{c}\text { Total } \\
\mathrm{mv}(-) \\
(\mathrm{gm} \mathrm{km} / \mathrm{s})\end{array}$ & $\begin{array}{c}\text { Exp. } \\
\mathrm{mv}(-) \\
(\mathrm{gm} \mathrm{km} / \mathrm{s})\end{array}$ & $\begin{array}{c}\text { Target } \\
\mathrm{mv}(+) \\
(\mathrm{gm} \mathrm{km} / \mathrm{s})\end{array}$ & $\begin{array}{c}\text { Projectile } \\
\mathrm{mv}(+) \\
(\mathrm{gm} \mathrm{km} / \mathrm{s})\end{array}$ & $\begin{array}{c}\text { Total } \\
\mathrm{mv}(+) \\
(\mathrm{gm} \mathrm{km} / \mathrm{s})\end{array}$ & $\begin{array}{c}\text { Exp. } \\
\mathrm{mv}(+) \\
(\mathrm{gm} \mathrm{km} / \mathrm{s})\end{array}$ \\
\hline \hline 8 & 0.2 & 5.4 & 5.6 & 3.88 & 9.6 & 12.7 & 22.3 & 27.61 \\
\hline 9 & 0.3 & 8.5 & 8.8 & 9.43 & 14.6 & 19.8 & 34.4 & 37.06 \\
\hline 10 & 0.1 & 10.0 & 10.1 & 10.38 & 17.0 & 23.6 & 40.6 & 53.76 \\
\hline 11 & 0.2 & 11.5 & 11.7 & 12.33 & 19.5 & 26.7 & 46.2 & 54.55 \\
\hline
\end{tabular}

Test 9 (Figure 7) consists of a shell of evenly distributed debris particles that are much smaller than the width of the debris cloud with a somewhat high density region at the leading surface of the cloud. In this case, the leading edge of the cloud has two distinct regions (see A in Figure 7) but they are less pronounced than in Test 8. The front-most $6 \mathrm{~cm}$ of the cloud has a somewhat higher density than the tail. Figure 11 displays the $\mathrm{CTH}$ results for Test 9 in axi-symmetric geometry using the same display technique as noted above. Inspection of the results reveals that the smaller radius debris bubble is composed of material from the thin sheet and the remainder of the debris cloud is composed of projectile material. Even though the debris cloud diameter is under-predicted by $\mathrm{CTH}$, the general mass distribution (in particular, the dual radius debris structure at the leading edge) is matched by the simulations. The debris cloud in Test 9 is still composed of solid fragments although the fragment sizes are much smaller. For Test 9, the material distribution is more accurately modelled by the continuum approximations of hydrocodes than Test 8 .

Test 10 (Figure 8) is more difficult to analyze due to a relatively low resolution radiograph. Figure 12 displays the computational linear density and shows that $\mathrm{CTH}$ predicts the width of the debris cloud quite closely $(\sim 4 \%)$ at that time. The shape of the debris cloud again displays the characteristic double radii at the leading edge and CTH matches the feature qualitatively. Interrogation of the CTH results indicate that the debris is composed mainly of molten droplets with possibly some vapor. The leading edge has a slightly higher density, as is replicated by the CTH simulations.

Test 11 (Figure 9) consists of a half shell of diffuse molten/vapor fragments with a distinctly higher density region at the leading edge of the cloud. In this case, the leading edge of the cloud does not have two distinct regions as in previous experiments. Very diffuse tendrils of material trail back from the leading half shell. The experimental debris cloud is very characteristic of hypervelocity debris clouds where 
phase changes have taken place. Figure 13 displays the computational linear density and shows that CTH again predicts the width of the debris cloud quite closely $(-4 \%)$. As with Test 10 , the general mass distribution is matched by the simulations. The diffuse tendrils trailing from the leading half shell of the debris cloud cannot be represented by the axi-symmetric geometry used for this study. However, alternate visualizations show that the mass in the tail of the debris cloud is confined to a shell at the outer edge of the cloud as shown in Figure 13. The mass is composed of small molten/vaporized fragments, uniformly distributed in a shell, and consists of high temperature material that could be vaporous.

The debris cloud size comparison data is summarized in Table 3. One can see that the predictions of debris cloud size for those impacts where fragmentation is a dominant phenomena are low by $\sim 20-30 \%$ and that the predictions for those impacts where phase change is more important are low by only $\sim 4 \%$. This would lead one to believe that for this particular experimental-computational series, the principal source of error is material constitutive behavior and not the equation of state.

The next principal point of comparison has to do with the transfer of momentum along the initial direction of motion of the projectile. The momentum in the forward $(\mathrm{mv}(+)$ in the table) and rearward (mv(-) in the table) direction was measured experimentally by ballistic pendulum. The experimental data is compared to the CTH predictions in Table 4. Considering the accuracy of the experimental data (the actual error was not reported in the literature, but could be $+/-10 \%$ or more), the agreement between the predicted and experimental results is excellent. CTH consistently under-predicts both forward and rearward momentum with one exception. The error is relatively constant as the velocity increases, $\sim 4 \%$ for the rearward and $\sim 20 \%$ for the forward momentum.

In general, the agreement between CTH and the experimental data is good. The principal source of discrepancy between $\mathrm{CTH}$ and the data is in the prediction of fragmentation in relatively low velocity impacts. Some of these differences could be due to the simple constitutive (linear-elastic, perfectly-plastic) and failure $\left(\mathrm{P}_{\min }\right.$ with void insertion) models used in the calculations. The choice of models was made for two reasons; first, we intended to focus the modelling on the high velocity regime where material constitutive behavior was not as important; and, second, little is known about the details of the constitutive and failure behavior of zinc at high strain rates and pressures. We feel that the agreement is acceptable given the sophistication of the material models. Depending on the physical phenomena being investigated, this study indicates that hydrocodes can be used to predict debris distribution and momentum transfer accurately and can give some direction to the integrated stress levels that a secondary target might experience.

\section{REFERENCES}

1. Mullin, S. A., C. E. Anderson, Jr., J. S. Wilbeck, and D. K. Apo Dissimilar Material Velocity Scaling for Hypervelocity Impact, Proceedings of the 1989 Hypervelocity Impact Symposium (1990).

2. T. G. Trucano Equation of State and Fragmentation Issues in Computational Lethality Analysis, Sandia National Laboratories Report SAND92-2397 (1993).

3. Anderson, Jr., C. E., E. S. Hertel, Jr., S. A. Mullin, and R. L. Hunt, The Hypervelocity Experimental Research Database (HERD): Support for the Defense Nuclear Agency Code Validation Program, to appear in the 1994 AIAA Space Programs and Technologies Conference (1994).

4. Hertel, Jr., E. S., R. L. Bell, M. G. Elrick, A. V. Farnsworth, G. I. Kerley, J. M. McGlaun, S. V. Petney, S. A. Silling, P. A. Taylor, and L. Yarrington, CTH: A Software Family for Multi-Dimensional Shock Physics Analysis, Proceedings of the 1993 International Symposium on Shock Waves (1994).

5. Chhabildas, L. C. and E. S. Hertel, Jr., Comparison of Analytic Whipple Bumper Shield Ballistic Limits with CTH Simulations, Sandia National Laboratories Report SAND92-0347 (1992).

6. Hertel, Jr., E. S., Simulations of Orbital Debris Impacts on Bumper Shields, Proceedings of the First European Conference on Space Debris (1993).

7. Wise, J. L., G. I. Kerley, and T. G. Trucano, Shock-Vaporization Studies on Zinc and Porous Carbon, in Shock Waves in Condensed Matter-1991, edited by S. C. Schmidt, J. W. Jorbes, and R. D. Dick, Elsevier Science Publishers B. V. (1992).

\section{ACKNOWLEDGEMENTS}

The authors would like to thank Scott Mullin of Southwest Research Institute and Mark Smith of AEDC in their help in obtaining high quality reproductions the original experimental data.

This work performed at Sandia National Laboratories supported by the U. S. Department of Energy under contract DE-AC04-94AL85000. Funding for the simulations was provided by the Wright Laboratory. 